\title{
Compliance with dietary guidelines in the Spanish population. Results from the Catalan Nutrition Survey
}

\author{
Lluís Serra-Majem ${ }^{1,2 *}$, Lourdes Ribas ${ }^{2}$ and Josep M. Ramon ${ }^{2}$ \\ ${ }^{1}$ University of Las Palmas de Gran Canaria, Departamento de Ciencias Clínicas, Facultad de Medicina y Ciencias de la \\ Salud, Apartado de Correos 550, 35080 Las Palmas de Gran Canaria, Spain \\ ${ }^{2}$ Catalan Nutrition Survey Group, Community Nutrition Research Unit, Scientific Park of Barcelona, University of Barcelona, \\ Av. Dr Gregorio Maranon s/n, 08028 Barcelona, Spain
}

\begin{abstract}
The aim of the present study was to analyse the different food and nutrition profiles of the section of the Spanish population having either a low or a high intake of energy, fat, saturated fatty acids (SFA) and fibre in order to analyse the compliance with dietary guidelines. Analyses were made from the Catalan Nutrition Survey, comprising a random sample of 1600 people aged 18 to 60 . Dietary information was obtained by means of two $24 \mathrm{~h}$ recall, and food and energy intake was categorized according to the quartiles of fat, SFA and fibre intake. The results show the food consumption patterns that distinguish low versus high fat, SFA and fibre eaters in the Spanish population, and facilitate the establishment of food-based dietary guidelines in Spain and other Mediterranean countries.
\end{abstract}

Food consumption: Nutrition survey: Dietary guidelines: Spain

Spain can be considered a typical Mediterranean country that enjoys the so-called 'Mediterranean Diet'. The term 'Mediterranean Diet' has become popular in recent years, and has entered the language not only of the general public but also of scientific publications, with consistently positive, albeit rather vague, connotations. However, the term is used loosely and for varying purposes, and uncertainties remain about the dietary pattern it applies to, where in the Mediterranean region such a diet is to be found, and precisely what it is expected to do for health (Ferro-Luzzi \& Sette, 1989; Keys, 1995; Nestle, 1995). More recently, the major characteristics of this dietary pattern have been defined (Helsing \& Trichopoulou, 1989; Serra-Majem \& Helsing, 1993) as have its time trends and its potential use as a dietary guide for non-Mediterranean countries (James et al. 1989; Nestle, 1995; Willett et al. 1995).

Like the other southern European countries, Spain has no formal nutrition policy, but the Ministries of Agriculture and Health conduct many related activities (MAPA, 1991). The major sources of information about food availability and dietary intake in Spain are: Food Balance Sheets, Household Budget Surveys conducted by the National Institute of Statistics every 10 years, Household Consumption Surveys conducted yearly by the Ministry of Agriculture from 1987, and individual dietary surveys at regional level: (Canary Islands (1983-1985), Catalonia (1986), Basque Country
(1989), Murcia (1990), Madrid (1992-1993), Catalonia (1992-1993), Valencia (1993-1994), Andalusia (19961997), Canary Islands (1997-1998) and Spain, infant and adolescents (1998-ongoing). Despite these many sources of information, there is no coordinated food consumption monitoring system at the national level. Furthermore, the periodic evaluations of Spain's health objectives do not probe nutrition indicators, except for obesity, which is estimated using self-reported weight and height (Regidor et al. 1993; Serra-Majem et al. 1993).

With the exception of Malta, no European Mediterranean country has a national nutrition policy. Most countries have developed nutrition objectives and dietary guidelines as well as some system for monitoring nutritional information, but no structured nutritional policies exist. Several reasons explain this gap. Nutrition receives scant attention from the public health sector, perhaps because it is less relevant as a risk factor for chronic disease in the Mediterranean than in northern Europe. The role of the European Union in agricultural policies also affects development of nutrition policies. Another reason is the less interventionist attitude toward health policy in the Mediterranean. Finally, there is great emphasis placed on consumer demand in formulating nutritional policy in Mediterranean countries (SerraMajem et al. 1997). In Spain, the 17 autonomous regions are responsible for development of health plans consistent with

*Corresponding author: Professor Lluís Serra-Majem, fax +34 928 453475, email 1serra@ cicei.ulpgc.es 
the World Health Organization Regional Office for Europe (WHO-Europe) 'Health for All for the Year 2000' initiative and with the Spanish health law. To date, ten regions have reinforced their health plans with a law or decree, and only four have not yet approved one. All the regions have been empowered by central government to control health promotion and health protection, except those concerning international health, but only six have been empowered to control health care planning and administration (Catalonia was empowered earlier, in 1982, five years before the Basque Country, Valencia and Andalusia) (MSC, 1994). Nearly all of the regional health plans have included an analysis of the situation, health objectives, and describe specific programmes and activities to be developed. Some regional health plans do not include nutritional objectives, even though nutritional information from household budget surveys can be desegregated at the regional level. In addition, recent nutritional surveys have been conducted or are ongoing in several regions, and surveys were also conducted in the Canary Islands and in Catalonia 10 years ago (Doreste, 1987; Aranceta et al. 1990, 1994; Violan et al. 1991; Vioque \& Quiles, 1995; Serra-Majem et al, 1996). However, since 1995, no nutritional and dietary guidelines have been defined for the Spanish State.

Present-day scientific and epidemiological knowledge points to a clearly defined relation between consumption of fat and various high-profile chronic diseases, which has led a group of $\mathrm{FAO} / \mathrm{WHO}$ experts to recommend that a maximum fat intake of $30 \%$ energy be set as the population goal. Very low-fat diets can cause problems in satisfying energy and nutrient needs. This group, therefore, suggested that the lower limit for fat intake should be set at
$15 \%$ energy, a sufficient level for covering needs for essential fatty acids and fat-soluble vitamins. The group, like many other bodies, recommended distributing the energy intake evenly between SFA (10\%), monounsaturated fatty acids (MUFA) (10\%) and polyunsaturated fatty acids (PUFA) (10\%), though no scientific criteria were established, except with SFA $(<10 \%)$. In Spain, as in the other Mediterranean countries, these general WHO recommendations are difficult to implement. In our diet, the contribution of olive oil represents between 13 and $20 \%$ of consumed energy (according to regions), which makes it difficult to carry out the plan to reduce the contribution of energy from fat to $30 \%$. In the context of the Mediterranean diet it would be possible to maintain the present level of olive oil consumption and aim at reducing the energy provided by SFA by $3-4 \%$.

Data from the latest food surveys carried out in Spain, generally, put the energy intake from fats at between 37 and $42 \%$. Though it involved modifying the WHO recommendations, the Spanish Association for Community Nutrition developed a set of nutritional recommendations with the assent of the Nutrition Unit of the WHO Regional Office for Europe. In Table 1, these are compared with the WHO recommendations and an estimate of the present situation in Spain. These recommendations were developed at a meeting on 24 October 1994 during a Consensus Conference held in Barcelona. The main point was to recommend a fat intake below $35 \%$ energy if olive oil is the most important fat consumed (as is the case in most of the Spanish regions). The proposal to design a dietary guide for the Spanish population in a pyramidal chromatic structure was also discussed and debated at the consensus meeting.

Table 1. Nutritional recommendations for Europe (WHO-Europe) and Spain (Spanish Association of Community Nutrition)

\begin{tabular}{|c|c|c|c|}
\hline & $\begin{array}{l}\text { Estimated } \\
\text { present situation } \\
\text { in Spain* }\end{array}$ & $\begin{array}{c}\text { Nutritional } \\
\text { Objectives } \\
\text { (WHO) }\end{array}$ & $\begin{array}{l}\text { Nutritional } \\
\text { Objectives } \\
\text { (SENC) } ¥\end{array}$ \\
\hline Body weight & $\mathrm{BMI}=25-26$ & BMI 20-25 & BMI 20-25 \\
\hline Total fat (\%energy) & $40 \S$ & $20-30$ & $\begin{array}{l}\leq 35 \| \\
\leq 309\end{array}$ \\
\hline SFA (\%energy) & 13 & 10 & $\leq 10$ \\
\hline PUFA (\%energy) & 7 & $\mathrm{PUFA} / \mathrm{SFA}=1$ & $\begin{array}{l}\text { UF/SF } \geq 2 \cdot 0 \\
\text { PUFA } \leq 8\end{array}$ \\
\hline Cholesterol (mg/1000 Kcal) & 150 & $<100$ & $\leq 100$ \\
\hline Sugar (\%energy) & 10 & 10 & $\leq 10$ \\
\hline Complex carbohydrate (\%energy) & 33 & $45-55$ & $>50$ \\
\hline Fibre $(g / d)$ & $\leq 20$ & 30 & $>25$ \\
\hline Nutrient density & Acceptable & & \\
\hline Salt $(g / d)$ & 9 & 5 & $<6$ \\
\hline Proteins (\%energy) & $>15$ & $12-13$ & 13 \\
\hline Alcohol & $6 \%$ energy & Limit & $\begin{array}{c}\text { Reduce } \leq 1-2 \text { glasses } \\
\text { of wine/day }\end{array}$ \\
\hline $\begin{array}{l}\text { Fluorides in water }(\mathrm{mg} / \mathrm{l}) \\
\text { lodine prophylaxis }\end{array}$ & $\begin{array}{c}<0.3 \\
\text { variable }\end{array}$ & $\begin{array}{l}0.7-1 \cdot 2 \\
\text { included }\end{array}$ & fluoridated-iodinated salt \\
\hline
\end{tabular}

${ }^{*}$ Adapted data from different studies.

†From WHO-Europe 1987 (James, 1989).

¥ $\mathrm{SENC}=$ Spanish Association of Community Nutrition

Guidelines adopted in a Consensus Conference held in Barcelona (Serra-Majem et al. 1995).

$\S$ Alcohol-free energy.

$\|$ Frequent use of olive oil.

INot using olive oil frequently.

SFA = Saturated fatty acids; PUFA = Polyunsaturated fatty acids; UF/SF $=$ Unsaturated fats/saturated fats; BMI = Body Mass Index. 
The aims of the present study were to analyse the different food and nutritional profiles of the sections of the Catalan population having either a low or a high intake of energy, fat, SFA and fibre.

\section{Material and methods}

The present analysis was performed using the database of the Catalan Nutritional Survey (1992-93); the methodology of this survey has been described elsewhere (Serra-Majem et al. 1996) and will be summarized here. The random sample population consisted of inhabitants aged 6 to 75 living in Catalan municipalities. The sample was stratified according to the results of the household budget survey (HBS) and randomized into subgroupings with Catalan municipalities being the primary sample units, and individuals within these municipalities comprising the final sample units. The theoretical sample size was estimated to be 3000 subjects. This would provide for a specific relative precision between 7 and $10 \%(\alpha=0.05)$ for those estimates between 10 and $20 \%$.

The sample was obtained from the census registers of the 82 randomly selected municipalities. The sample distribution according to stratification by the HBS was as follows: 46 municipalities with fewer than 10000 inhabitants, 28 municipalities with more than 10000 but fewer than 100000 inhabitants, and 8 municipalities consisting of more than 100000 inhabitants. Samples were selected by considering the proportion of people in, and the specific weight of, each municipality in the sample, with the aim of avoiding, as far as possible, further requirements for sample weighting.

The dietary assessment consisted of combining two $24 \mathrm{~h}$ recalls and a food frequency questionnaire covering 77 food items. The $24 \mathrm{~h}$ recall was carried out twice during the 1992 study period, the first in a warm season (May-July) and the second in a cold season (November-December). This was to avoid the influence of seasonal variations. The surveys were always carried out at the home of the subject being interviewed. There were 36 interviewers, who were assigned an average of 78 subjects each, which allowed the fieldwork to be completed within the 6-week timeframe anticipated for this phase of the project. The interviewers underwent a rigorous selection process and training programme, thus establishing a quality-control mechanism that lasted throughout the entire period of the dietary data collection. Twenty percent of the sample were interviewed by telephone in order to verify some aspects of the dietary information previously gathered by personal interview. The coding of food data was carried out by the same interviewers, under the supervision of two dieticians.

In order to estimate the volumes and portion sizes, the household measures found in the subjects' own homes were used. Volumes for these measures were also noted. On some occasions standard portion sizes were used. Diet histories for subjects with disabilities or memory defects were carried out via the primary caretaker. Conversion of food into nutrients was made by the French 'Répertoire générale des aliments', which comprises 572 foods specifying 32 nutrients, energy and edible portion (Feinberg et al. 1991). Adjustment for intra-individual variability was made by analysis of variance, using the method described by Beaton and Liu (Liu et al. 1978; Beaton et al, 1983).

For the present analysis the population aged 18 to 60 was distributed according to their quartile of fat, SFA and fibre intake, using adjusted values for intra-individual variability. The consumption of main food groups was compared between low and high consumers (first and fourth quartile) of fat, SFA and fibre intake. Comparison also included percentage of energy intake from fat, carbohydrate and protein. Averages were always standardized by age using the indirect method.

\section{Results and discussion}

Out of a total of 4000 individuals included in the random sample, $2757(68.9 \%)$ participated in the first interview (May-July) and 2475 (61.9\%) people in the second. Nonparticipation rates included potential subjects declining to be interviewed as well as involuntary non-participation due to census error caused by address changes, missing persons or unavoidable impediments to survey collaboration. Of

Table 2. Daily intakes of energy, fat, SFA, fibre and fruit and vegetables by Catalan subjects in 1992 (aged 18 to 60 years)

\begin{tabular}{|c|c|c|c|c|c|c|}
\hline \multirow[b]{2}{*}{ Nutrients and foods } & \multicolumn{2}{|c|}{$\begin{array}{c}\text { Men } \\
(n=712)\end{array}$} & \multicolumn{2}{|c|}{$\begin{array}{c}\text { Women } \\
(n=888)\end{array}$} & \multicolumn{2}{|c|}{$\begin{array}{c}\text { Total } \\
(n=1600)\end{array}$} \\
\hline & Mean & (SD) & Mean & (SD) & Mean & (SD) \\
\hline Energy (MJ) & $10 \cdot 3$ & 1.3 & $7 \cdot 7$ & 1.3 & 8.9 & 1.8 \\
\hline Total fat (\%energy) & 37.5 & 3.4 & 38.4 & 4.0 & 38.0 & 3.8 \\
\hline SFA (\%energy) & 12.5 & 1.8 & $13 \cdot 0$ & $2 \cdot 0$ & $12 \cdot 8$ & 1.9 \\
\hline MUFA (\%energy) & $17 \cdot 2$ & 1.9 & $17 \cdot 2$ & 1.9 & $17 \cdot 2$ & 1.9 \\
\hline PUFA (\%energy) & 4.6 & 0.8 & 4.6 & 0.8 & $4 \cdot 6$ & 0.8 \\
\hline Protein (\%energy) & $18 \cdot 7$ & $2 \cdot 0$ & $20 \cdot 2$ & $3 \cdot 3$ & $19 \cdot 6$ & 2.9 \\
\hline Carbohydrate (\%energy) & 39.9 & 2.5 & 40.5 & 4.2 & $40 \cdot 2$ & 3.6 \\
\hline Alcohol (\%energy) & 4.3 & 4.2 & $1 \cdot 2$ & 1.9 & $2 \cdot 6$ & 3.5 \\
\hline Alcohol (g) & 14.8 & 14.9 & 3.2 & $5 \cdot 0$ & 8.4 & $12 \cdot 1$ \\
\hline Fibre $(\mathrm{g})$ & $18 \cdot 6$ & 3.2 & 15.5 & $2 \cdot 1$ & $16 \cdot 8$ & $3 \cdot 1$ \\
\hline Fibre/MJ energy (g/MJ) & 1.81 & 0.3 & $2 \cdot 1$ & 0.4 & 1.9 & 0.4 \\
\hline Fruit and vegetables $(\mathrm{g})$ & 455 & 288 & 500 & 259 & 480 & 270 \\
\hline
\end{tabular}

Data adjusted by intra-individual variability and age standardized.

SFA = saturated fatty acids; MUFA = monounsaturated fatty acids; PUFA = polyunsaturated fatty acids 
these 2475 people, 1600 individuals were between the ages of 18 and 60 years, and were included in the present analysis. Our data have been adjusted by intra-individual variability and the age standardized; crude data from two
$24 \mathrm{~h}$ recall questionnaires do not allow the estimation of nutrient-intake distribution among populations, and age adjustment is needed since food consumption and nutrient intake is largely influenced by age.

Table 3. Mean daily nutrient and food intakes of Catalan subjects aged 18 to 60 years in the lowest $\left(Q_{1}\right)$ and highest $\left(Q_{4}\right)$ quartiles of fat intake

\begin{tabular}{|c|c|c|c|c|c|c|c|c|}
\hline & \multicolumn{4}{|c|}{ Men $(n=712)$} & \multicolumn{4}{|c|}{ Women $(n=888)$} \\
\hline $\begin{array}{l}\text { Energy (MJ) } \\
\text { Protein (\%energy) } \\
\text { Carbohydrates (\%energy) } \\
\text { Alcohol (\%energy) } \\
\text { Total fat (\%energy) } \\
\text { SFA (\%energy) } \\
\text { MUFA (\%energy) } \\
\text { PUFA (\%energy) } \\
\text { Alcohol (g) } \\
\text { Fibre (g) } \\
\text { Fibre (g/MJ) }\end{array}$ & & $\begin{array}{r}9 \cdot 3 \\
19 \cdot 4 \\
40 \cdot 2 \\
6 \cdot 9 \\
33 \cdot 2 \\
10 \cdot 7 \\
15 \cdot 1 \\
4 \cdot 2 \\
22 \cdot 2 \\
18 \cdot 8 \\
2 \cdot 0\end{array}$ & & $\begin{array}{r}10 \cdot 2 \\
18 \cdot 7 \\
38 \cdot 0 \\
3.5 \\
41.1 \\
13.8 \\
19.1 \\
5.1 \\
11.9 \\
17.9 \\
1.8\end{array}$ & & $\begin{array}{r}7 \cdot 2 \\
21 \cdot 3 \\
44 \cdot 3 \\
1 \cdot 4 \\
33 \cdot 2 \\
10 \cdot 9 \\
15 \cdot 1 \\
4 \cdot 0 \\
3 \cdot 5 \\
16 \cdot 1 \\
2 \cdot 3\end{array}$ & & $\begin{array}{r}8.1 \\
19.2 \\
36 \cdot 9 \\
1.0 \\
43.3 \\
14.9 \\
19.3 \\
5 \cdot 0 \\
2.7 \\
14.9 \\
1.9\end{array}$ \\
\hline $\begin{array}{l}\text { Potatoes } \\
\text { Fried potatoes }\end{array}$ & $\begin{array}{l}73 \\
34\end{array}$ & $\begin{array}{l}75 \\
24\end{array}$ & $\begin{array}{l}81 \\
49\end{array}$ & $\begin{array}{l}93 \\
46\end{array}$ & $\begin{array}{l}65 \\
19\end{array}$ & $\begin{array}{l}60 \\
10\end{array}$ & $\begin{array}{l}77 \\
42\end{array}$ & $\begin{array}{l}80 \\
32\end{array}$ \\
\hline $\begin{array}{l}\text { Dairy foods } \\
\text { Full-fat milk } \\
\text { Other milk } \\
\text { Yogurts } \\
\text { Cheese }\end{array}$ & $\begin{array}{l}93 \\
68 \\
19 \\
18 \\
46\end{array}$ & $\begin{array}{r}188 \\
96 \\
35 \\
23 \\
14\end{array}$ & $\begin{array}{l}98 \\
76 \\
11 \\
26 \\
58\end{array}$ & $\begin{array}{r}205 \\
121 \\
14 \\
23 \\
25\end{array}$ & $\begin{array}{l}97 \\
63 \\
43 \\
37 \\
40\end{array}$ & $\begin{array}{r}228 \\
94 \\
74 \\
38 \\
12\end{array}$ & $\begin{array}{l}99 \\
80 \\
31 \\
31 \\
67\end{array}$ & $\begin{array}{r}259 \\
144 \\
43 \\
28 \\
24\end{array}$ \\
\hline $\begin{array}{l}\text { Meat/fish/poultry/egg } \\
\text { Fresh meat } \\
\text { Processed meat } \\
\text { Poultry } \\
\text { Fish } \\
\text { Eggs }\end{array}$ & $\begin{array}{l}99 \\
85 \\
75 \\
53 \\
75 \\
67\end{array}$ & $\begin{array}{r}297 \\
81 \\
35 \\
55 \\
98 \\
27\end{array}$ & $\begin{array}{r}100 \\
90 \\
82 \\
45 \\
63 \\
80\end{array}$ & $\begin{array}{r}336 \\
122 \\
54 \\
46 \\
71 \\
43\end{array}$ & $\begin{array}{r}100 \\
74 \\
63 \\
59 \\
69 \\
46\end{array}$ & $\begin{array}{r}227 \\
60 \\
20 \\
60 \\
70 \\
16\end{array}$ & $\begin{array}{r}100 \\
86 \\
77 \\
45 \\
59 \\
86\end{array}$ & $\begin{array}{r}255 \\
93 \\
34 \\
43 \\
48 \\
37\end{array}$ \\
\hline Pulses (cooked) & 36 & 34 & 21 & 18 & 25 & 21 & 25 & 20 \\
\hline $\begin{array}{l}\text { Fats } \\
\text { MUFA (olive oil) } \\
\text { PUFA oils } \\
\text { Butter, lard, margarine }\end{array}$ & $\begin{array}{r}100 \\
99 \\
82 \\
22\end{array}$ & $\begin{array}{r}27 \\
21 \\
4 \\
1\end{array}$ & $\begin{array}{r}100 \\
100 \\
89 \\
39\end{array}$ & $\begin{array}{r}44 \\
34 \\
7 \\
2\end{array}$ & $\begin{array}{r}100 \\
98 \\
68 \\
25\end{array}$ & $\begin{array}{r}20 \\
15 \\
2 \\
2\end{array}$ & $\begin{array}{r}100 \\
100 \\
91 \\
52\end{array}$ & $\begin{array}{r}38 \\
29 \\
5 \\
4\end{array}$ \\
\hline Confectionery & 83 & 18 & 85 & 21 & 79 & 17 & 20 & 16 \\
\hline Nuts & 12 & 3 & 15 & 5 & 8 & 2 & 14 & 3 \\
\hline $\begin{array}{l}\text { Drinks }(\mathrm{ml}) \\
\text { Tea } \\
\text { Coffee } \\
\text { Drinking chocolate } \\
\text { Soft drinks }\end{array}$ & $\begin{array}{r}1 \\
87 \\
4 \\
34\end{array}$ & $\begin{array}{r}1 \\
86 \\
6 \\
63\end{array}$ & $\begin{array}{r}2 \\
83 \\
1 \\
30\end{array}$ & $\begin{array}{r}2 \\
72 \\
2 \\
63\end{array}$ & $\begin{array}{r}6 \\
78 \\
5 \\
26\end{array}$ & $\begin{array}{r}9 \\
63 \\
4 \\
50\end{array}$ & $\begin{array}{r}7 \\
81 \\
5 \\
26\end{array}$ & $\begin{array}{r}11 \\
71 \\
6 \\
56\end{array}$ \\
\hline
\end{tabular}

$* \%$ Cons. $=\%$ Consumers.

Data adjusted by intra-individual variability and age standardized.

SFA = saturated fatty acids; PUFA = polyunsaturated fatty acids; MUFA = monounsaturated fatty acids. 
Table 4. Mean daily nutrient and food intakes of Catalan subjects aged 18 to 60 years in the lowest $\left(Q_{1}\right)$ and highest $\left(Q_{4}\right)$ quartiles of SFA intake

\begin{tabular}{|c|c|c|c|c|c|c|c|c|}
\hline & \multicolumn{4}{|c|}{ Men $(n=712)$} & \multicolumn{4}{|c|}{ Women $(n=888)$} \\
\hline $\begin{array}{l}\text { Energy (MJ) } \\
\text { Protein (\%energy) } \\
\text { Carbohydrates (\%energy) } \\
\text { Alcohol (\%energy) } \\
\text { Total fat (\%energy) } \\
\text { SFA (\%energy) } \\
\text { MUFA (\%energy) } \\
\text { PUFA (\%energy) } \\
\text { Alcohol (g) } \\
\text { Fibre (g) } \\
\text { Fibre (g/MJ) }\end{array}$ & & $\begin{array}{r}9 \cdot 6 \\
19 \cdot 2 \\
40 \cdot 3 \\
6 \cdot 7 \\
34 \cdot 2 \\
10 \cdot 2 \\
15 \cdot 8 \\
4.5 \\
21.9 \\
19 \cdot 0 \\
2 \cdot 0\end{array}$ & & $\begin{array}{r}10 \cdot 8 \\
18 \cdot 3 \\
39 \cdot 4 \\
2 \cdot 6 \\
40.0 \\
14.7 \\
18 \cdot 2 \\
4.6 \\
9.5 \\
17 \cdot 8 \\
1.7\end{array}$ & & $\begin{array}{r}7 \cdot 1 \\
21 \cdot 7 \\
43 \cdot 0 \\
1 \cdot 5 \\
34 \cdot 2 \\
10 \cdot 4 \\
15 \cdot 7 \\
4 \cdot 5 \\
3 \cdot 6 \\
16 \cdot 1 \\
2 \cdot 3\end{array}$ & & $\begin{array}{r}8 \cdot 1 \\
19 \cdot 3 \\
37.9 \\
1.2 \\
42.0 \\
15 \cdot 6 \\
18.3 \\
4.5 \\
3.3 \\
14.5 \\
1.8\end{array}$ \\
\hline $\begin{array}{l}\text { Potatoes } \\
\text { Fried potatoes }\end{array}$ & $\begin{array}{l}78 \\
37\end{array}$ & $\begin{array}{l}83 \\
32\end{array}$ & $\begin{array}{l}81 \\
45\end{array}$ & $\begin{array}{l}81 \\
37\end{array}$ & $\begin{array}{l}67 \\
22\end{array}$ & $\begin{array}{l}61 \\
15\end{array}$ & $\begin{array}{l}72 \\
36\end{array}$ & $\begin{array}{l}67 \\
23\end{array}$ \\
\hline $\begin{array}{l}\text { Dairy foods } \\
\text { Full-fat milk }\end{array}$ & $\begin{array}{l}90 \\
56\end{array}$ & $\begin{array}{r}155 \\
72\end{array}$ & $\begin{array}{l}99 \\
85\end{array}$ & $\begin{array}{l}301 \\
196\end{array}$ & $\begin{array}{l}95 \\
51\end{array}$ & $\begin{array}{r}205 \\
60\end{array}$ & $\begin{array}{l}99 \\
88\end{array}$ & $\begin{array}{r}287 \\
17\end{array}$ \\
\hline $\begin{array}{l}\text { Other milk } \\
\text { Yogurts } \\
\text { Cheese }\end{array}$ & $\begin{array}{l}20 \\
23 \\
34\end{array}$ & $\begin{array}{r}36 \\
25 \\
9\end{array}$ & $\begin{array}{l}10 \\
33 \\
71\end{array}$ & $\begin{array}{l}12 \\
30 \\
33\end{array}$ & $\begin{array}{l}53 \\
35 \\
33\end{array}$ & $\begin{array}{l}93 \\
33 \\
10\end{array}$ & $\begin{array}{l}29 \\
27 \\
77\end{array}$ & $\begin{array}{l}36 \\
24 \\
30\end{array}$ \\
\hline $\begin{array}{l}\text { Meat/fish/poultry/egg } \\
\text { Fresh meat } \\
\text { Processed meat } \\
\text { Poultry } \\
\text { Fish } \\
\text { Eggs }\end{array}$ & $\begin{array}{l}99 \\
82 \\
73 \\
55 \\
80 \\
67\end{array}$ & $\begin{array}{r}307 \\
73 \\
34 \\
61 \\
111 \\
29\end{array}$ & $\begin{array}{r}100 \\
94 \\
89 \\
44 \\
53 \\
76\end{array}$ & $\begin{array}{r}311 \\
125 \\
58 \\
44 \\
46 \\
38\end{array}$ & $\begin{array}{l}99 \\
71 \\
57 \\
60 \\
78 \\
52\end{array}$ & $\begin{array}{r}241 \\
54 \\
17 \\
64 \\
87 \\
18\end{array}$ & $\begin{array}{r}100 \\
86 \\
81 \\
42 \\
59 \\
79\end{array}$ & $\begin{array}{r}246 \\
93 \\
37 \\
39 \\
44 \\
32\end{array}$ \\
\hline Pulses (cooked) & 31 & 29 & 22 & 17 & 20 & 17 & 23 & 17 \\
\hline $\begin{array}{l}\text { Fats } \\
\text { MUFA (olive oil) } \\
\text { PUFA oils } \\
\text { Butter, lard, margarine }\end{array}$ & $\begin{array}{r}100 \\
99 \\
81 \\
17\end{array}$ & $\begin{array}{r}31 \\
25 \\
5 \\
1\end{array}$ & $\begin{array}{r}100 \\
99 \\
88 \\
52\end{array}$ & $\begin{array}{r}37 \\
27 \\
5 \\
4\end{array}$ & $\begin{array}{r}100 \\
98 \\
73 \\
18\end{array}$ & $\begin{array}{r}25 \\
19 \\
3 \\
1\end{array}$ & $\begin{array}{r}100 \\
100 \\
90 \\
64\end{array}$ & $\begin{array}{r}32 \\
22 \\
4 \\
5\end{array}$ \\
\hline Confectionery & 79 & 21 & 87 & 26 & 77 & 15 & 85 & 20 \\
\hline Nuts & 14 & 3 & 12 & 3 & 9 & 2 & 11 & 2 \\
\hline $\begin{array}{l}\text { Drinks }(\mathrm{ml}) \\
\text { Tea } \\
\text { Coffee } \\
\text { Drinking chocolate } \\
\text { Soft drinks }\end{array}$ & $\begin{array}{r}2 \\
81 \\
2 \\
37\end{array}$ & $\begin{array}{r}1 \\
78 \\
5 \\
82\end{array}$ & $\begin{array}{r}3 \\
76 \\
6 \\
46\end{array}$ & $\begin{array}{r}4 \\
65 \\
7 \\
98\end{array}$ & $\begin{array}{r}6 \\
77 \\
4 \\
22\end{array}$ & $\begin{array}{r}8 \\
65 \\
3 \\
42\end{array}$ & $\begin{array}{r}7 \\
82 \\
7 \\
35\end{array}$ & $\begin{array}{r}9 \\
72 \\
10 \\
73\end{array}$ \\
\hline
\end{tabular}

*\% Cons. $=\%$ Consumers.

Data adjusted by intra-individual variability and age standardized.

$\mathrm{SFA}=$ saturated fatty acids; PUFA = polyunsaturated fatty acids; MUFA = monounsaturated fatty acids. 
Table 2 shows the mean intakes of energy, fat, SFA, fibre, alcohol and fruit and vegetables in the Catalan population aged 18 to 60 years. Total fat represents $38 \%$ of total energy intake, and SFA $12.8 \%$; both these percentages were higher in women than in men. Protein represents almost $20 \%$ energy and alcohol $2.6 \%$ energy. The consumption of fruit and vegetables was $480 \mathrm{~g}$ (455 among men and 500 among women). In both sexes, the first quartile for relative fat

Table 5. Mean daily nutrient and food intakes of Catalan subjects aged 18 to 60 years in the lowest $\left(Q_{1}\right)$ and highest $\left(Q_{4}\right)$ quartiles of fibre intake

\begin{tabular}{|c|c|c|c|c|c|c|c|c|}
\hline & \multicolumn{4}{|c|}{ Men $(n=712)$} & \multicolumn{4}{|c|}{ Women $(n=888)$} \\
\hline $\begin{array}{l}\text { Energy (MJ) } \\
\text { Protein (\%energy) } \\
\text { Carbohydrates (\%energy) } \\
\text { Alcohol (\%energy) } \\
\text { Total fat (\%energy) } \\
\text { SFA (\%energy) } \\
\text { MUFA (\%energy) } \\
\text { PUFA (\%energy) } \\
\text { Alcohol (g) } \\
\text { Fibre (g) } \\
\text { Fibre (g/MJ) }\end{array}$ & & $\begin{array}{r}10 \cdot 7 \\
18 \cdot 8 \\
39 \cdot 2 \\
4 \cdot 7 \\
38 \cdot 7 \\
13 \cdot 4 \\
17 \cdot 7 \\
4 \cdot 5 \\
16 \cdot 9 \\
16 \cdot 3 \\
1.5\end{array}$ & & $\begin{array}{r}9.2 \\
19 \cdot 4 \\
40 \cdot 0 \\
4.6 \\
35.8 \\
11.3 \\
16 \cdot 4 \\
4.6 \\
14.6 \\
20.9 \\
2.3\end{array}$ & & $\begin{array}{r}9.0 \\
18.9 \\
39.8 \\
1.2 \\
40.3 \\
14.2 \\
17.8 \\
4.7 \\
3.6 \\
14.3 \\
1.6\end{array}$ & & $\begin{array}{r}6 \cdot 9 \\
21 \cdot 2 \\
41 \cdot 9 \\
1 \cdot 1 \\
36 \cdot 0 \\
11 \cdot 6 \\
16 \cdot 3 \\
4.4 \\
2.6 \\
17 \cdot 2 \\
2.5\end{array}$ \\
\hline $\begin{array}{l}\text { Potatoes } \\
\text { Fried potatoes }\end{array}$ & $\begin{array}{l}72 \\
42\end{array}$ & $\begin{array}{l}77 \\
40\end{array}$ & $\begin{array}{l}75 \\
36\end{array}$ & $\begin{array}{l}75 \\
26\end{array}$ & $\begin{array}{l}76 \\
46\end{array}$ & $\begin{array}{l}77 \\
33\end{array}$ & $\begin{array}{l}71 \\
20\end{array}$ & $\begin{array}{l}64 \\
10\end{array}$ \\
\hline $\begin{array}{l}\text { Dairy foods } \\
\text { Full-fat milk } \\
\text { Other milk } \\
\text { Yogurts } \\
\text { Cheese }\end{array}$ & $\begin{array}{l}97 \\
81 \\
11 \\
28 \\
57\end{array}$ & $\begin{array}{r}255 \\
162 \\
16 \\
26 \\
24\end{array}$ & $\begin{array}{l}92 \\
66 \\
20 \\
23 \\
50\end{array}$ & $\begin{array}{r}197 \\
102 \\
34 \\
26 \\
19\end{array}$ & $\begin{array}{l}99 \\
86 \\
19 \\
31 \\
62\end{array}$ & $\begin{array}{r}287 \\
179 \\
35 \\
27 \\
21\end{array}$ & $\begin{array}{l}96 \\
59 \\
51 \\
39 \\
51\end{array}$ & $\begin{array}{r}223 \\
75 \\
82 \\
37 \\
19\end{array}$ \\
\hline $\begin{array}{l}\text { Meat/fish/poultry/egg } \\
\text { Fresh meat } \\
\text { Processed meat } \\
\text { Poultry } \\
\text { Fish } \\
\text { Eggs }\end{array}$ & $\begin{array}{r}100 \\
90 \\
83 \\
55 \\
62 \\
68\end{array}$ & $\begin{array}{r}341 \\
116 \\
55 \\
66 \\
71 \\
34\end{array}$ & $\begin{array}{l}99 \\
85 \\
72 \\
51 \\
73 \\
66\end{array}$ & $\begin{array}{r}288 \\
83 \\
35 \\
60 \\
81 \\
32\end{array}$ & $\begin{array}{r}100 \\
87 \\
85 \\
50 \\
67 \\
73\end{array}$ & $\begin{array}{r}283 \\
92 \\
39 \\
55 \\
70 \\
26\end{array}$ & $\begin{array}{l}99 \\
76 \\
62 \\
46 \\
65 \\
57\end{array}$ & $\begin{array}{r}218 \\
68 \\
19 \\
45 \\
64 \\
22\end{array}$ \\
\hline Pulses (cooked) & 10 & 6 & 38 & 38 & 17 & 11 & 29 & 28 \\
\hline $\begin{array}{l}\text { Fats } \\
\text { MUFA (olive oil) } \\
\text { PUFA oils } \\
\text { Butter, lard, margarine }\end{array}$ & $\begin{array}{r}100 \\
100 \\
84 \\
34\end{array}$ & $\begin{array}{r}33 \\
26 \\
5 \\
2\end{array}$ & $\begin{array}{r}100 \\
99 \\
83 \\
27\end{array}$ & $\begin{array}{r}33 \\
26 \\
5 \\
2\end{array}$ & $\begin{array}{r}100 \\
100 \\
89 \\
52\end{array}$ & $\begin{array}{r}34 \\
25 \\
5 \\
4\end{array}$ & $\begin{array}{r}100 \\
99 \\
70 \\
31\end{array}$ & $\begin{array}{r}26 \\
19 \\
3 \\
2\end{array}$ \\
\hline Confectionery & 88 & 26 & 84 & 20 & 89 & 25 & 74 & 14 \\
\hline Nuts & 8 & 2 & 17 & 5 & 14 & 3 & 11 & 2 \\
\hline $\begin{array}{l}\text { Drinks }(\mathrm{ml}) \\
\text { Tea } \\
\text { Coffee } \\
\text { Drinking chocolate } \\
\text { Soft drinks }\end{array}$ & $\begin{array}{r}1 \\
83 \\
4 \\
55\end{array}$ & $\begin{array}{r}1 \\
78 \\
4 \\
140\end{array}$ & $\begin{array}{r}2 \\
81 \\
3 \\
24\end{array}$ & $\begin{array}{r}5 \\
67 \\
7 \\
44\end{array}$ & $\begin{array}{r}6 \\
73 \\
7 \\
48\end{array}$ & $\begin{array}{r}8 \\
59 \\
11 \\
101\end{array}$ & $\begin{array}{r}7 \\
76 \\
3 \\
15\end{array}$ & $\begin{array}{r}8 \\
73 \\
2 \\
25\end{array}$ \\
\hline
\end{tabular}

* $\%$ Cons. $=\%$ Consumers.

Data adjusted by intra-individual variability and age standardized.

SFA = saturated fatty acids; PUFA = polyunsaturated fatty acids; MUFA = monounsaturated fatty acids. 
consumption was $36 \%$ energy, and that of SFA was $10 \cdot 9$ \%energy both close to the Spanish recommendations (SerraMajem et al. 1995; Sera-Majem \& Aranceta, 1997). For the 4th quartile (high consumers), the cut-off point was $42.6 \%$ energy for total fat and $14.4 \%$ energy for SFA.

Table 3 shows the energy from macronutrients and foodgroup intake among low and high consumers of fat. Differences in consumption levels with fat intake (low versus high quartile) are evident for fried potatoes, yoghurts, butter, cheese, eggs and fresh and processed meat, which are higher among high-fat consumers in both sexes. On the other hand, intakes of pulses, poultry and fish are higher among low-fat eaters. Marked differences can be seen in the average intake of fruits (particularly non-citrus fruits), which is much greater among low-fat consumers. This is not so for vegetable consumption, which appears to be greater among the high-fat eaters. In addition, the average intakes in the highfat group compared to the low-fat group are higher for edible fats, pastries, whole milk, cheese, fresh and processed meat and eggs, and lower for pulses, coffee, cereals (particularly bread), low-fat milk and fish. These results will allow public health authorities to improve nutrition interventions aiming at increasing or decreasing the percentage of consumers and/or the total amount consumed of the different food groups. Regarding nutrient intakes, the differences are obviously important for energy, total fat and SFA, and made low-fat consumers show a lower intake of alcohol $(22.2 \mathrm{~g}$ ) versus $11.9 \mathrm{~g}$ in the higher-fat group). These results do not support the idea that heavy eaters (high fat, energy intake) drink more alcohol, and they increase our understanding of alcohol consumption behaviour in the Mediterranean region.

Table 4 shows the results according to the intake of SFA. Men in the low-SFA group have an average intake of $10.2 \%$ energy, and women of $10.4 \%$ energy, and the highSFA group averages are $14.7 \%$ energy and $15.6 \%$ energy respectively. The same differences in energy, total fat and alcohol observed in Table 3 are also observed in this table. There are two kinds of differences between the groups: the percentage of consumers and average intakes; the low-SFA group have a higher percentage of consumers of citrus and other fruits, pulses, pasta, low-fat milk, poultry and fish for men, and of citrus and other fruits, rice, low-fat milk, poultry and fish for women. The percentage of consumers in the same group is lower for fried potatoes, pastries, whole milk, yogurts, cheese, soft drinks, butter, fruit juice (only in men) and PUFA oils (particularly in women). The differences in average intakes are much greater in the lower group of SFA: vegetables, pulses (only in men), poultry and fish (the total meat group remains the same), and much smaller for soft drinks, pastries, dairy products, fresh and processed meat, and eggs.

Table 5 shows differences in food and nutrient intake according to fibre intake. Average intakes in the low-fibre group are $1.5 \mathrm{~g} / \mathrm{MJ}$ for men and 1.6 for women, and in the high-fibre group are 2.3 for men and $2.5 \mathrm{~g} / \mathrm{MJ}$ for women. Differences in fruit and vegetables consumption are very marked, since the average is $245 \mathrm{~g}$ in the lower group and $671 \mathrm{~g}$ in the higher group for men and $345 \mathrm{~g}$ and $698 \mathrm{~g}$ for women. The percentage of energy from fat and SFA is slightly higher among people consuming less fibre. The most important differences in food group consumption in the high-fibre group are a markedly higher intake of fruit and vegetables (more than the double that observed in the low-fibre group) and pulses (more than three times low-fibre group consumption), and a low consumption of soft drinks, cereals, particularly pastries, fried potatoes (particularly in women), dairy products and all meats and fish. Intake of fat and other foods remains similar in both groups. Cereal group consumption does not determine fibre intake in our population, which is mainly influenced by the consumption of fruit, vegetables and pulses; in addition,the percentage of people eating wholegrain cereal products is still very low.

The results of the present analysis indicate the dietary interventions that need to be considered for the Catalan population, emphasizing those interventions that appear to have priority.

\section{Acknowledgements}

This study was supported by contract between the Health Department of the Autonomous Government of Catalonia and the University of Barcelona Bosch Gimpera Foundation.

The Catalan Nutrition Survey Group comprised: Lluís Serra-Majem (Director), Lourdes Ribas (coordinator), Reina García-Closas (coordinator), Josep M. Ramon, Gemma Salvador, Andreu Farran, Jaume Serra, Glòria Sabater, Lluís Jover, Ricard Tresserras, Esteve Saltó, Pilar Chacón, $\mathrm{M}^{\mathrm{a}}$ Cruz Pastor, Joan Fernández-Ballart, Gonçal Lloveras, Josep Lluis Taberner, Lluís Salleras.

\section{References}

Aranceta J, Pérez C, Equileor I, González de Galdeano L, Mataix J \& Saez de Buruaga J (1990) Encuesta Nutricional de País Vasco (Basque Country Nutritional Survey). Vitoria: Gobierna Vasco.

Aranceta J, Pérez C, Amela C \& García R (1994) Encuesta de nutricion de la communidad de Madrid. Madrid: Consejeria de salud de la comunidad de Madrid.

Beaton GH, Miller J, McGuire V, Feather TE \& Little JA (1983) Sources of variance in 24-hour dietary recall data: implications for nutrition study design and interpretation. Carbohydrate sources, vitamins and minerals. American Journal of Clinical Nutrition 37, 986-995.

Doreste JL (1987) Encuesta de alimentación y valoración nutricional de la comunidad canaria (Dietary survey and nutritional assessment of the Canarian Community). PhD Thesis, Universidad de la Laguna.

Feinberg M, Favier JC \& Ireland-Ripert J (1991) Répertoire général des aliments. Paris: Tec \& Doc Lavoisier.

Ferro-Luzzi A \& Sette S (1989) The Mediterranean diet: an attempt to define its present and past composition. European Journal of Clinical Nutrition 43 (suppl. 2), 13-30.

Helsing E \& Trichopoulou A [editors] (1989) The Mediterranean Diet and Food Culture: a Symposium. European Journal of Clinical Nutrition 43 (suppl. 2), 1-92.

James WPT, Duthie GG \& Wahle KWJ (1989) The Mediterranean diet: protective or simply non-toxic? European Journal of Clinical Nutrition 43 (suppl. 2), 31-42.

Keys A (1995) Mediterranean diet and public health: personal reflections. American Journal of Clinical Nutrition $\mathbf{6 1}$ (suppl.), 1321S-1323S.

Liu K, Stamler J, Dyer A, Mckeever J \& McKeever P (1978) 
Statistical methods to assess and minimize the role of intraindividual variability in obscuring the relationship between dietary lipids and serum cholesterol. Journal of Chronic Diseases 31, 399-418.

MAPA (Ministerio de Agricultura Pesca y Alimentación) (1991) Consumo alimentario en España 1990 (Food consumption in Spain, 1990, Tomos I y II. Madrid: Dirección General de Política Alimentaria.

MSC (Ministerio de Sanidad y Consumo) Dirección General de Alta Inspección y Relaciones Institucionales (1994) Estudio descriptivo de los Planes de Salud en España (Descriptive study of the health plans in Spain). Madrid: Mimeo.

Nestle M (1995) Mediterranean diets: historical and research overview. American Journal of Clinical Nutrition 61 (suppl.), 1313S-1320S.

Regidor E, Gutiérrez JL \& Rodríguez C (1993) Indicadores de salud. Segunda evaluación en España del programa regional europeo salud para todos (Health indicators. Second Spanish evaluation of the Regional European programme on health for all). Madrid: Ministerio de Sanidad y Consumo, 143-144.

Serra-Majem L \& Aranceta J on behalf of the working group on dietary guidelines of the Spanish association of community nutrition (1997) Nutrition and dietary guidelines for the Spanish population. Tool for a nutrition policy in Spain. In Implementing Dietary Guidelines, pp. 233-244 [V Wheelock, editor]. London: Chapman \& Hall.

Serra-Majem L \& Helsing E [editors] (1993) Changing patterns of fat intake in Mediterranean Countries. European Journal of Clinical Nutrition 47 (suppl. 1), 1-100.

Serra-Majem L, Ribas L, Lloveras G \& Salleras L (1993) Changing patterns of fat consumption in Spain. European Journal of Clinical Nutrition 47 (suppl.), S13-S20.

Serra-Majem L, Aranceta J \& Mataix J [editors] (1995) Documento de consenso. Guías Alimentarias para la población española (Consensus document. Dietary guidelines for the Spanish population). Barcelona: SG-Editores.

Serra-Majem L, Ribas L, García-Closas R, Ramon JM, Salvador G, Farran A, Serra J, Sabater G, Jover L, Tresserras R, Salto E, Chacon P, Pastor MC, Puchal A, Lloveras G, Taberner JL \& Salleras L (1996) Llibre blanc: Avaluació de l'estat nutricional de la població catalana (1992-93) (White book: The evaluation of nutritional status in Catalonia, Spain (1992-93)). Barcelona: Departament de Sanitat i Seguretat Social.

Serra-Majem L, Ferro-Luzzi A, Bellizzi MC \& Salleras L (1997) Nutrition policies in Mediterranean Europe. Nutrition Reviews 55 (suppl. II), S39-S54.

Violan C, Stevens L \& Molina F (1991) Encuesta de alimentación en la población adulta de Murcia 1990 (Dietary survey of the Murcia population 1990). Murcia: Consejería de Sanidad, Dirección General de Salud. Serie informes no 7.

Vioque J \& Quiles J (1995) Resultados preliminares de la encuesta de Nutrición de la Comunidad valenciana (preliminary results of the Valencia survey). In Documento de consenso: guías alimentarias para la población española, pp. 121-124 [L Serra Majem, J Aranceta and J Mataix, editors] Barcelona: Sociedad Española de Nutrición Comunitaria-SG Editores.

Willett WC, Sacks F, Trichopoulou A, Drescher G, Ferro-Luzzi A, Helsing E \& Trichopoulos D (1995) Mediterranean diet pyramid: a cultural model for healthy eating. American Journal of Clinical Nutrition 61 (suppl.), 1402S-1406S. 